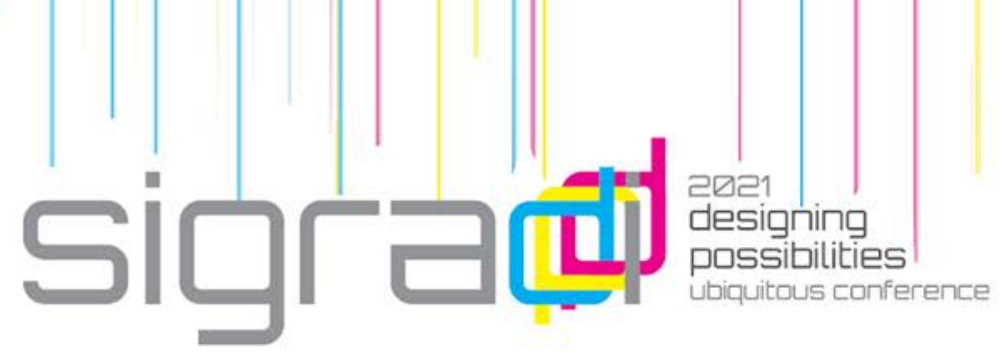

\title{
Non-Fungible Building Components: Using Smart Contracts for a Circular Economy in the Built Environment
}

\author{
Theodoros Dounas ${ }^{1}$, Wassim Jabi $^{2}$, Davide Lombardi ${ }^{3}$ \\ ${ }^{1}$ Robert Gordon University, UK \\ t.dounas@rgu.ac.uk \\ ${ }^{2}$ Cardiff, University, UK \\ JabiW@cardiff.ac.uk \\ ${ }^{3}$ Xi'an Jiaotong Liverpool University, China \\ davide.lombardi@xjtlu.edu.cn
}

\begin{abstract}
The presented research study tackles the topic of economic and material sustainable development in the built environment and construction industry by introducing and applying the concept and the potential of Non-Fungible Tokens (NFTs) on blockchain within the early stages of the design process via the interface of common design software. We present a digital infrastructure layer for architectural assets and building components that can integrate with AEC supply chains, enabling a more effective and articulated development of circular economies. The infrastructure layer consists of a combination of topology graphs secured with a blockchain. The paper concludes with a discussion about the possibilities of material passports as well as circular economy and smart contracts as an infrastructure for whole lifecycle BIM and digital encapsulation of value in architectural design.
\end{abstract}

Keywords: Non-fungible tokens, Blockchain, Supply Chain, Topology, Circular Economy

\section{Introduction \& Problem Statement}

The Construction Industry is a wasteful sector and has a negative impact on carbon footprint, both on operations and as embodied carbon [Anderson et al., Pomponi et al.]. Part of the problem in terms of waste and the circular economy begins with the lack of incentive to design for the end of life of a building [Adams et al.] but also, on a practical basis, the lack of information to re-use components and materials [reference]. To re-use materials from one building that is demolished or taken apart to the next, the concept of material passports 
has been invented, with the idea that a material cadastre needs to be created so that the involved stakeholders are able to track materials over the lifecycle of a building [Heisel et al.]. This paper primarily examines a procedure to set up a material and building components cadastre, providing a computational strategy to implement it relying on blockchain technologies. Secondarily, we sketch out a potential solution to the problem of incentives for designers so that their design approach allows for the creation of a complete circular economy of buildings.

\subsection{Background and State of the Industry}

Low carbon materials and efficiencies in the operation of buildings are considered as two of the mitigating solutions to the emissions of greenhouse gases that contribute to climate change. [IPCC report, chapter 4]. "Built environment is responsible for significant use of energy (62\%) and is a major source of greenhouse emissions (55\%)" [Anderson et al.]. Embodied carbon and carbon from operational use are the dominant factors in the environmental impact of a built asset, with construction surprisingly contributing for only $2 \%$. Embodied carbon and operational carbon are interrelated: decreasing one increases the other. Significant gains in both are possible with optimised architectural designs. In parallel, carbon efficiencies are interconnected with supply chain structure, which in turn is integral to the social and economic aspects of the Architecture-Engineering-Construction (AEC) industry, making the carbon impact of the built environment a difficult problem to solve. As such, an interdisciplinary collaboration framework that allows AEC stakeholders to create improved carbon mitigation and adaptation strategies is needed [Pomponi et al.]. Such a collaboration framework may be thought to exist within the Building Information Modelling (BIM) paradigm; however, the paradigm has so far not delivered radical improvements in carbon reductions. The complexity of the models being used, lack of interdependence and data on supply chains, lack of stakeholders' engagement, and non-availability of carbon performance data in the full lifecycle analysis have led to a reduced impact on carbon reduction by those technologies advocating them. Potentially, one effective solution to the problem of embodied carbon and reduced waste is the introduction of a circular economy that enables the recycling of buildings.

However, that introduces the second problem regarding the information related to buildings' components and materials, as well as the provenance and retrieval of that information over the lifecycle of the building. Norouzi et al. have developed an extensive bibliographical analysis of the dimensions of circular economy in the past 20 years of research, and through that, identified that issues of circular economy in buildings relate to the introduction of circular business models and the development of smart cities and industry 4.0. Earlier, Adams et al., have identified fragmentation of the construction industry as an issue at the core of any circular business or operational model, while mentioning that the lack of market mechanisms to aid recovery of materials is 
one of the top challenges for stakeholders in the built environment when attempting to implement circular economy projects. Along with a fragmented supply chain, they also identified the lack of technical information and incentives as two significant barriers to adopting of circular economy practices in the design and whole lifecycle of the built environment assets. A crucial aspect that is essentially needed for the creation of a circular economy is the material passports: structured information attached to a particular material that communicates the traceability of the material (i.e where it came from and where it is located in buildings) its properties, its use, and the best way to extract the material from the building. Additionally, it can include a manual of deconstruction to ensure the recovery on the materials and their value.

\subsection{Blockchain: State Machine}

Blockchains are a type of decentralised computing infrastructure running on a variety of network nodes that act as Turing complete State machines [Antonopoulos et al.]. They have been used initially for cryptocurrency transactions; however, with the expansion of their ability to run smart contracts, classes of software that execute automatically without human intervention, blockchains have advanced into acting as decentralised computers. Due to the nature of their security and computational structure [EthHash algorithm], public blockchains have the following characteristics:

1. Data on the blockchain is immutable after a block's finality, i.e the end of the transaction and the confirmation by all computing nodes.

2. Participation in the blockchain itself and in acting in the "right" manner is incentivized via token acquisition, where the token holds some value

3. Resiliency: as the state machine is run in a myriad of nodes, loss of information is improbable, as we would need to lose all nodes to lose the information. As such, blockchains are excellent for the permanent storage of certain types of information.

4. Traceability: immutability and permanence give rise to traceability, i.e, data provenance on the blockchain, where everything can be traced in a transparent manner.

The ability of a blockchain to also run smart contracts gives rise to the ability to create fungible and non-fungible tokens. Fungible tokens are the same and exchangeable, while Non-Fungible Tokens [reference retracted] (NFTs) are unique digital objects that are created and tracked through a smart contract on a blockchain, such as Ethereum. In essence, an NFT is a registration place for a unique data object that can reside either on the blockchain itself or on a similar decentralised data storage system such as the Interplanetary Filesystem (IPFS). Within IPFS, content is not retrieved based on its address (where?) but by the nature of the content itself (what?). This is achieved by producing a hash 
contract for NFTs written in solidity, and IPFS for storing all the additional metadata of the NFTs.

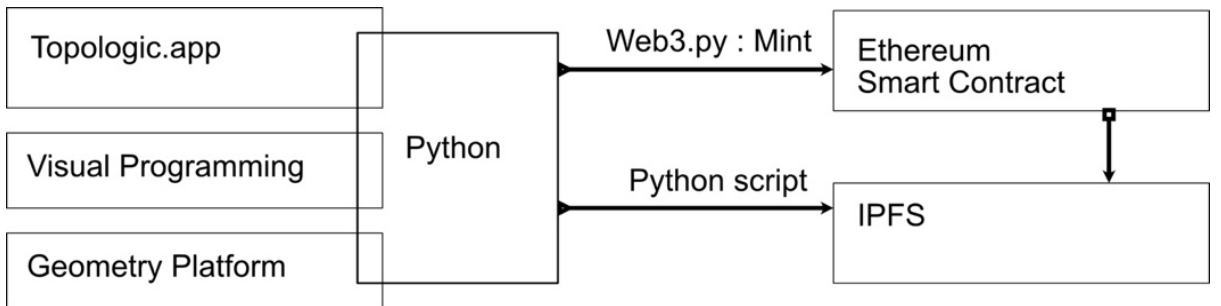

Figure 2. Interplay between Topologic, Smart Contract, and IPFS. Source: designed by Dounas, T., July 2021.

Topologic is a non-manifold Topology software library that has been invented to provide a rigorous toolkit to design software for architecture enabling the creation of nimble, lightweight models. It has been in development since 2017 and currently is available for Dynamo/Revit, Rhinoceros/Grasshopper add Blender/Sverchok. We use the blender version in the paper. The original first version of Topologic establishes a rigorous hierarchy of spatial constructs and topology entities [Jabi et al. 2018], starting from Cluster >CellComplex $>$ Cell $>$ Shell $>$ Face $>$ Wire $>$ Edge $>$ Vertex, where each class is parent to the next one. These are all contained into a super-class called Topology that additionally contains tools, methods, properties and constructors that in turn are used by the subclasses to extend it. Topologic is currently under active development, with a python wrapper making porting to python environments easier and extensions for the topologic classes that included Graphs, i.e., classes that represent the topological structural relationship between several topologies. Note that topologies are not interpreted directly into geometries, i.e a Topologic Edge can represent any line with complex curvature, or a Topologic Face can represent a surface with complex curvature. Once a Building is constructed via Topologic entities, we use a python language environment, the web3.py library and a custom python script to communicate the topology entities to the NFT Smart contract hosted at a particular address on the Ethereum Blockchain. The Topology entities are then encapsulated into "Topology String" entities, i.e strings that describe the topology itself, and as such, can be read by Topologic on any platform. The python script then triggers the creation of the Non-fungible token on the smart contract.

\subsection{Smart Contract}

The code for the contract is written in Solidity, the primary Ethereum smart contract language, and comes with the following features: it uses a counter to enumerate the NFTs, thus creating an easily identifiable number for each. It also sets the base Universal resource locater on IPFS, making it clear that we 


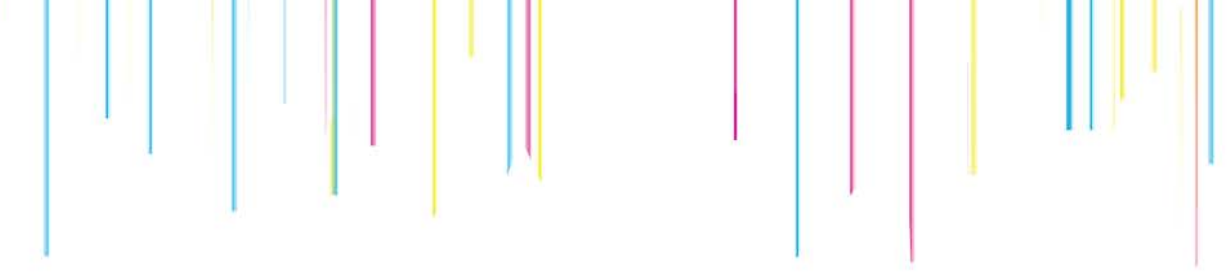

representing building components. In our case, we considered the vertical loadbearing elements made out of CLT; those are processed in Topologic to create a "Topology String" at the end, which, once fed into another Topologic script in a different platform, will recreate the same component without any loss of information. In parallel, the CLT component is represented inside Blender as an .ifc vertical load-bearing wall. The Industry Foundation Classes information is stored as metadata on IPFS and represents the material passport for this building component. Depending on the implementation of the required passport, additional information can be stored as metadata for the NFT of each component, related to the source and supply chain, its assembly and disassembly instructions and all related information needed for re-using the component as is, without recycling the material.

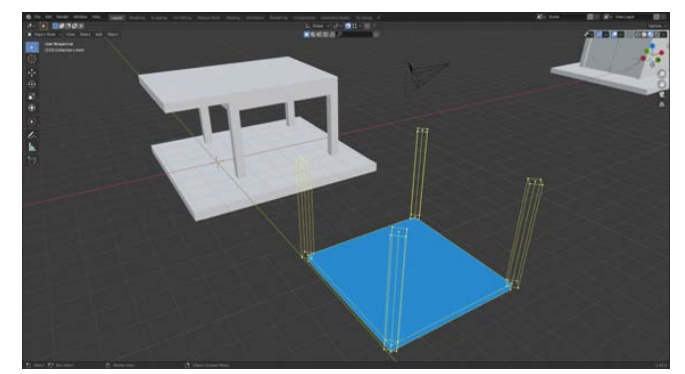

Figure 3. A Simple structure as described by Topologic on Blender. Source: setup and captured by Dounas, T. July 2021.

In parallel, the whole building is represented using a series of Topologic components through a dependency graph; thus, it has at its root in the whole building, followed by spatial entities (Cells), which are then interpreted as spatial enclosures. These enclosures are decomposed into faces and edges, which represents the building components. This dependency graph follows the rigorous logic of topologic analysis of non-Manifold Topology, and we use this file to mint the first NFT on the contract representing the whole building [Dounas et al. 2021] 


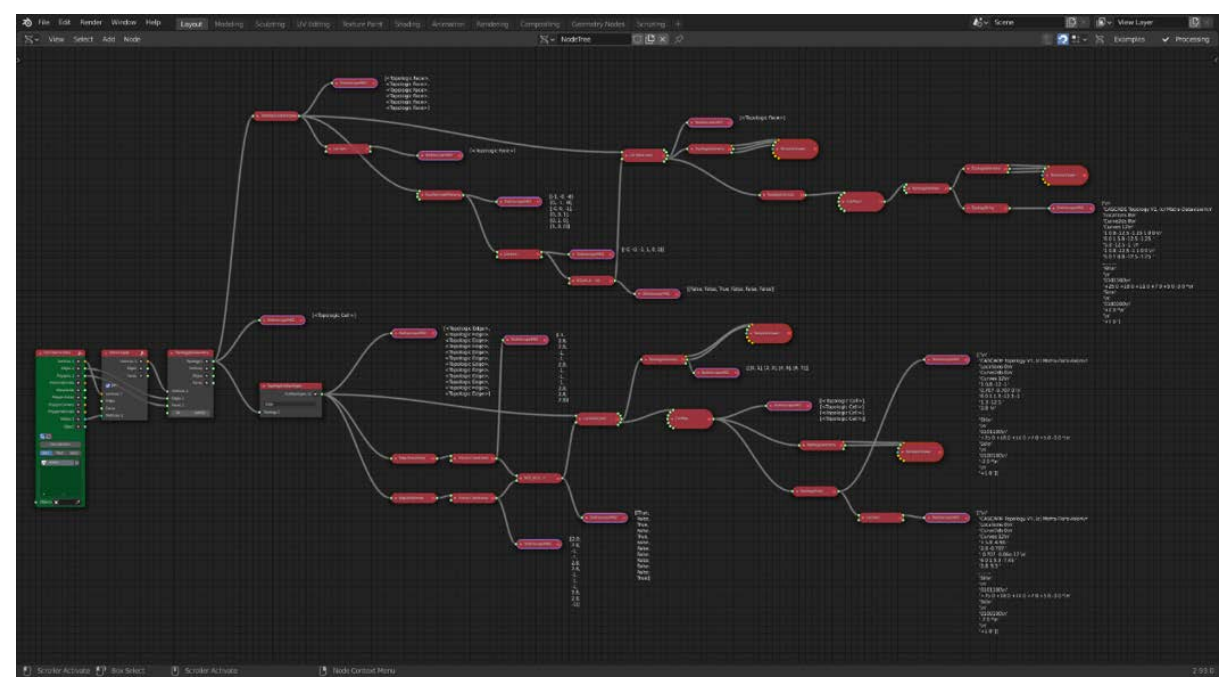

Figure 4. Topologic [on blender] - Representing a building component as a topology entity. Source: setup and captured by Dounas, T. \& Jabi, W. July 2021.

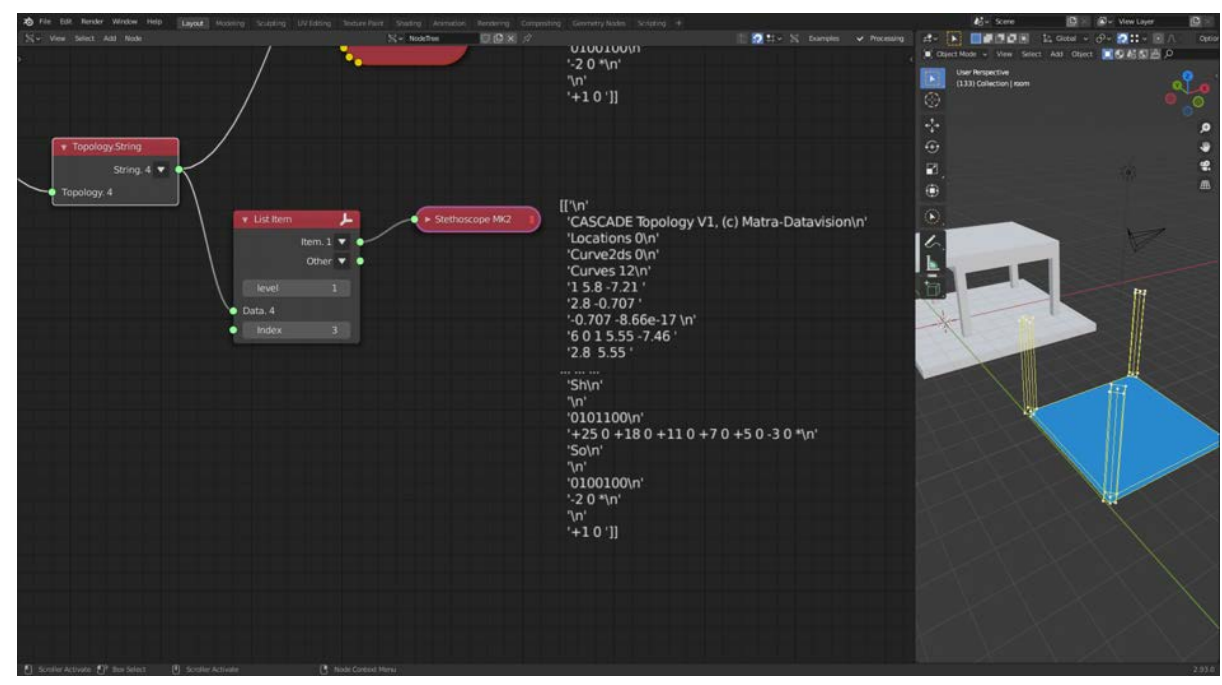

Figure 5. Topologic String and Component MetaData. Source: setup and captured by Dounas, T. \& Jabi, W. July 2021. 


\section{Smart Contracts and NFTs as a Material Cadastre for Buildings.}

We have demonstrated how to analyse a building into components that are interrelated topologically through Topologic; we then have demonstrated the production of Non-Fungible tokens that retain their interconnected topology and refer to material and IFC passport metadata on IPFS. Due to the resilient, longterm nature of the blockchain, these NFTs and our method have the potential to create a global, transparent cadastre and registry for material passports and building components.

This cadastre would potentially become an information infrastructure layer for a circular economy in the AEC industry if applied particularly in the field of Design for Manufacturing and Assembly, i.e. when the goal is to re-use building components rather than material recycling. For this to occur, the equivalent of a search engine would be necessary, as well as a waste and carbon calculator embedded into the design tools, so that architects designing new buildings would have immediate access to the components available for re-use at any particular time. These tools, working in context, would enable the treatment of existing buildings as material banks and shift their end of life from demolition and waste to re-use.

Further, the creation of NFTs out of building components allows the expansion of Digital Twins applications using blockchain technologies, where components are connected with particular aspects of the building performance and their maintenance potentially automated through smart contracts. Additionally, our method can help to enhance building cadastres, adding IFC information where needed.

However, what remains to explore is how to radically change the AEC industry using these information layers. More importantly, how to incentivise designers and built environments stakeholders to update their attitudes towards circular economies, a feat that would signal the radical shift of the AEC industry.

\section{References}

Antonopoulos et 2018, al. Mastering Ethereum, Building Smart contracts and dApps, O' Reilly, (Willey UK), 978-1491971949

Adams, K.T., Osmani, M., Thorpe, T., Thornback, J., 2017. Circular economy in construction: current awareness, challenges and enablers. Proceedings of the Institution of Civil Engineers - Waste and Resource Management 170, 15-24. https://doi.org/10.1680/jwarm.16.00011

Anderson, J. Wulfhorst, G. Lang, W. 2014, Energy analysis of the Built Environment-a review and outlook, in Renewable andf Sustainable Energy reviews journal, http://dx.doi.org/10.1016/j.rser.2014.12.027 pp149-158 
Benachio, G.L.F., Freitas, M. do C.D., Tavares, S.F., 2020. Circular economy in the construction industry: A systematic literature review. Journal of Cleaner Production 260, 121046. https://doi.org/10.1016/j.jclepro.2020.121046

Heisel, F., Rau-Oberhuber, S., 2020. Calculation and evaluation of circularity indicators for the built environment using the case studies of UMAR and Madaster. Journal of Cleaner Production 243, 118482. https://doi.org/10.1016/j.jclepro.2019.118482

Honic, M., Kovacic, I., Sibenik, G., Rechberger, H., 2019. Data- and stakeholder management framework for the implementation of BIM-based Material Passports. Journal of Building Engineering 23, 341-350. https://doi.org/10.1016/j.jobe.2019.01.017

Journal of Cleaner Production | ScienceDirect.com by Elsevier [WWW Document], n.d. URL https://www.sciencedirect.com/journal/journal-of-cleaner-production (accessed 7.19.21).

Norouzi, M., Chàfer, M., Cabeza, L.F., Jiménez, L., Boer, D., 2021. Circular economy in the building and construction sector: A scientific evolution analysis. Journal of Building Engineering 44, 102704. https://doi.org/10.1016/j.jobe.2021.102704

Pomponi, F. Moncaster, A. 2016. Embodied carbon mitigation and reduction in the built environment $\mathrm{e}$ What does the evidence say? Journal of Environmental management, vol 181, pp687-700

Dounas, T. Jabi, W. Lombardi, D. 2021. Topology Generated Non-Fungible Tokens Blockchain as infrastructure for a circular economy in architectural design, A. Globa, J. van Ameijde, A. Fingrut, N. Kim, T.T.S. Lo (eds.), PROJECTIONS - Proceedings of the 26th CAADRIA Conference - Volume 2, The Chinese University of Hong Kong and Online, Hong Kong, 29 March - 1 April 2021, pp. 151-160

Jabi, W.et al. 2018. Topologic: enhancing the representation of space in 3D modelling environments through non-manifold topology. Presented at: 36th annual Education and research in Computer Aided Architectural Design in Europe (eCAADe) 2018, Lodz, Poland, 17-21 September 2018.

Code repositories:

https://github.com/arlav/TopoToken

https://github.com/wassimj/topologicPy 